\title{
Core-shell PVA/gelatin nanofibrous scaffolds using co- solvent, aqueous electrospinning: Toward a green approach
}

\section{Mustafa Sengor}

Corresponding Author

E-mail address: mustafa.sengor@boun.edu.tr

Department of Mechanical Engineering, Faculty of Engineering, Bogazici University, , Bebek, Istanbul 34342, Turkey

Correspondence to: M. Sengor (E-mail: mustafa.sengor@boun.edu.tr

Alp Ozgun

Institute of Biomedical Engineering, Bogazici University, , Uskudar, Istanbul 34684, Turkey

Gulcan Corapcioglu

Faculty of Engineering and Natural Science, Sabanci University, , Tuzla, Istanbul 34956, Turkey

Mehmet Ipekoglu

Department of Mechatronic Systems Engineering, Faculty of Engineering, Turkish-German University, Beykoz, Istanbul 34820, Turkey

Bora Garipcan

Institute of Biomedical Engineering, Bogazici University, , Uskudar, Istanbul 34684, Turkey

Nuri Ersoy

Department of Mechanical Engineering, Faculty of Engineering, Bogazici University, , Bebek, Istanbul 34342, Turkey

Sabri Altintas

Department of Mechanical Engineering, Faculty of Engineering, Bogazici University, , Bebek, Istanbul 34342, Turkey 


\section{ABSTRACT}

Electrospinning (ES) of gelatin often requires cytotoxic organic solvents or acidic environments, which deteriorate cell recognition sites. In this study, aqueous, non-toxic, cosolvent ES was performed to obtain core-shell poly(vinyl alcohol) (PVA)/gelatin nanofiber scaffolds. Effects of the core/shell feed rate ratio (FRR) were investigated on a morphological and mechanical basis. PVA:gelatin ratio of 1:4 was the limiting ratio for specific voltage and electrode distance parameters to obtain uniform fibers. Core-shell bead-free structures were obtained at $8 \%$ PVA and gelatin aqueous solutions. A mean diameter of $280 \mathrm{~nm}$ was obtained for 1:1 FRR at $15 \mathrm{kV}$ and $15 \mathrm{~cm}$ of electrode distance. Crosslinking resulted in slight improvement in tensile strengths and severe decrease in ductility. Fourier transform infrared spectra revealed retention and improvement of stable secondary structures of gelatin after ES. The scaffolds almost degraded more than $60 \%$ in 14 days. Based on the results, present scaffolds hold great promise as suitable candidates for biomedical applications.

\section{INTRODUCTION}

Over the past few decades, there has been an expanding interest in biomedical scaffolds made of electrospun fibers in the field of tissue engineering. Scaffolds generally mimic the extracellular matrix of the targeted tissue in order to act as an interface from synthetic to natural tissue. They are mostly used in wound dressings, $\underline{1}-\underline{3}$ cartilage-bone, $\underline{4}$ cardio-vascular tissue engineering, $\underline{5}$ and in drug delivery applications. $\underline{6}, \underline{7}$

Electrospinning (ES) is a relatively simple and cost-effective method to produce nanofiber meshes with a wide range of materials (polymers, polymer-loaded ceramics, metals, and lipids). In a typical ES process shown in Figure 1 , fibers come out of a tubular cone by forcing a polymer solution through the fine tip of a needle into a region of electric field formed between the needle tip and a collector electrode, adhering on this electrode. With minor changes in the setup, many forms of surface and fiber morphologies like aligned, coreshell, porous, hollow, etc. fibers can be fabricated. $\underline{8}, \underline{9}$

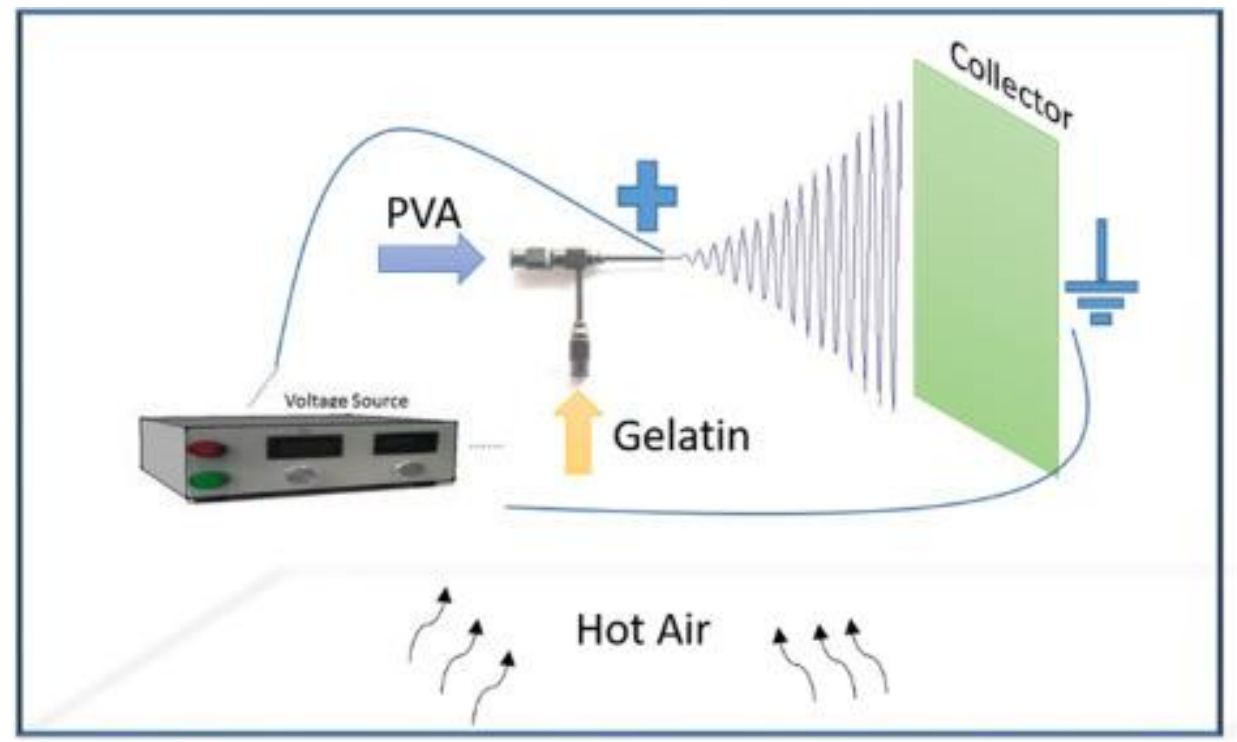

Figure 1 Schematic of the coaxial ES setup inside a closed chamber with a heating unit to produce PVA/gelatin core-shell electrospun fibers. 
To obtain fibers with a core-shell morphology, co-axial needles are needed and solutions are supplied from two different polymer sources with adjustable feed rates. This technique is commonly used in order to combine the power of natural polymers acting as recognition site with the power of synthetic polymers having superior mechanical endurance. Gelatin is one of the natural polymers widely used in tissue engineering having peptide domains for the recognition of integrin receptors of the cells. $10, \underline{11}$ Combining gelatin with a mechanically durable and biodegradable polymer such as polycaprolactone, poly(vinyl alcohol) (PVA), or chitin is a common practice in the literature. PVA-Gelatin is one of the most frequently used combinations. PVA, hydrolyzed form of poly(vinyl acetate), is a synthetic polymer known for being water soluble, biodegradable, and safe for human health.12-14

Organic polymers are usually spun by using highly volatile and acidic solvents, which are detrimental for human health. These processes also cause loss of conformational structure of natural polymers. 15 Furthermore, toxic residues remaining trapped inside these structures $\underline{16}$ present a potential risk. Using acidic environments as natural polymer solvents is an alternative but will result in partial degradation of the desired structure. 17 Another method is to use solvents like phosphate buffered saline (PBS), which has no toxic effect but requires desalination processes.

Since gelatin is soluble in water at around $40^{\circ} \mathrm{C}$, dissolving in aqueous solvents at elevated temperatures is one way to eliminate toxicity risks. In addition to this, aqueous PVA and gelatin form highly polar and immiscible solutions which make them potent candidates for coaxial ES with a stable flat interface between core and shell.18 However, it is important to note that gelatin can lose inherent alpha-helix structure above $40{ }^{\circ} \mathrm{C}$ after 4 h. 19

PVA-gelatin blends have been used to fabricate scaffolds in the literature. 20 Although this is an efficient method to aid cell adhesion and proliferation, it decreases the effect of gelatin due to response of cell to surface modifications. 21 In this study, gelatin was used as shell material to enhance first contact interaction with keeping mechanical advantages of PVA. Also in the literature, gelatin and PVA core-shell fibers were obtained by using PBS as solvent and ethanol inside the solution to arrange surface tension of solvents. 22 However, use of saltbased solvents require further desalination process and ethanol increases the risk of toxicity by integrating fibers constitution at the solution preparation stages. Also as in this study, use of a co-solvent method is an efficient method for preventing formation of defects, which arouse from different surface tensions of solvents.

Main purpose of this study is to develop a green approach to fabricate scaffolds that can be used in biomedical applications. Therefore, PVA/gelatin core-shell nanofiber scaffolds were fabricated in aqueous solvents by elevating spinning temperature up to $40{ }^{\circ} \mathrm{C}$ and using multinozzle system to increase spinning amount thereby decreasing time to conserve structure of gelatin. Morphologies of the sample surfaces and individual fibers were analyzed by scanning electron microscopy (SEM), transmission electron microscopy (TEM), and water contact angle measurements. Chemical structure was analyzed with Fourier transform infrared (FTIR) in order to detect material specific bands in samples. Differential scanning calorimeter (DSC) analysis was performed to characterize the thermal behavior of the nanofibers. Degradation of nanaofibers was evaluated by in vitro enzymatic degradation test. In the present study, water was the only solvent for both gelatin and PVA, resulting in smooth fiber surfaces. Thereby, possibility of a toxic effect believed to be reduced to unprecedented levels. 


\section{EXPERIMENTAL}

\section{Materials}

PVA (86\%-89\% hydrolyzed, average molecular weight $88,000 \mathrm{~g} / \mathrm{mol}$ ) was obtained from ZAG Chemicals (Istanbul, Turkey). Bovine Type-B gelatin (Bloom 200) was obtained from Merck (Darmstadt, Germany) in powder form. Glutaraldehyde $\left(25 \%\right.$ in $\left.\mathrm{H}_{2} \mathrm{O}\right)$, lysozyme, and PBS were purchased from Sigma Aldrich (Missouri, USA). Deionized water used throughout all ES experiments were obtained from Sigma Aldrich.

\section{Preparation of Polymer Solutions}

Aqueous PVA solutions ( $8 \% \mathrm{wt} / \mathrm{wt}$ ) were prepared first by charging PVA in deionized water slowly at ambient temperature, then allowing to swell for $10 \mathrm{~min}$ followed by heating up to $80^{\circ} \mathrm{C}$ and keeping solution at that temperature and concentration for about one hour. Gelatin aqueous solutions $(8 \% \mathrm{wt} / \mathrm{wt})$ were prepared by gently stirring gelatin for half an hour at $40{ }^{\circ} \mathrm{C}$ in deionized water.

\section{Electrospinning}

A custom-made multi-nozzle, temperature controlled setup was used. All of the experiments were conducted at $40{ }^{\circ} \mathrm{C}$ for $1 \mathrm{~h}$. A coaxial, horizontal setup was used with a core needle inner diameter of $0.9 \mathrm{~mm}$, outer diameter of $1.3 \mathrm{~mm}$, and a shell needle inner diameter of 1.6 $\mathrm{mm}$. Pure PVA (1P) and pure gelatin (1G) was spun as control samples with core needle at a feed rate of $0.1 \mathrm{~mL} / \mathrm{h}$ to compare with core-shell structure. A static collector wrapped with aluminum foil with dimensions of $10 \mathrm{~cm} \times 10 \mathrm{~cm}$ was used. Applied voltage, electrode distance, and relative humidity values were $15 \mathrm{kV}, 15 \mathrm{~cm}$, and $45 \%$, respectively. Four different feed rate ratios (FRRs) of PVA/gelatin were used for coaxial spinning: 4:1, 1:1, 1:2, and 1:4. These samples were named $4 \mathrm{P} 1 \mathrm{G}(0.4 \mathrm{~mL} / \mathrm{h}$ PVA: $0.1 \mathrm{~mL} / \mathrm{h}$ gelatin $), 1 \mathrm{P} 1 \mathrm{G}(0.1$ $\mathrm{mL} / \mathrm{h}$ PVA:0.1 mL/h gelatin $), 1 \mathrm{P} 2 \mathrm{G}(0.1 \mathrm{~mL} / \mathrm{h}$ PVA: $0.2 \mathrm{~mL} / \mathrm{h}$ gelatin $)$ and $1 \mathrm{P} 4 \mathrm{G}(0.1 \mathrm{~mL} / \mathrm{h}$ PVA:0.4 $\mathrm{mL} / \mathrm{h}$ gelatin), respectively. All samples were placed in a vacuum oven to eliminate remaining solvent.

\section{Crosslinking of Gelatin, PVA, and PVA-Gelatin Core-Shell Fibers}

Crosslinking procedure was conducted after extraction of fiber mats from aluminum foil by placing all fibers inside a sealed desiccator with $5 \% \mathrm{vol} / \mathrm{vol}$ glutaraldehyde in ethanol vapor for $24 \mathrm{~h}$ which was proven to be non-toxic at this level according to literature. $23, \underline{24}$

\section{Characterization}

Surface morphology of the nanofibers was imaged by SEM (Philips XL30) using a beam voltage of $10 \mathrm{kV}$. All samples were sputter-coated with Pt prior to SEM imaging. More than 100 randomly chosen fibers were examined by ImageJ software to determine the fiber diameter distribution. Average surface pore size of samples also analyzed with ImageJ software using at least 30 random pores from each group.

TEM analysis was performed with a JEOL-ARM 200 CFEG operating at $200 \mathrm{kV}$. Images were taken in STEM mode using HAADF detector. Fibers were cut into pieces and treated 
under potassium permanganate in ethanol solution for better visualization of the core structure. A drop of specimen was placed on carbon coated $300 \mu \mathrm{m}$ mesh size $\mathrm{Cu}$ grid. The grid was air dried at room temperature before loading on the microscope.

Chemical structure of pure PVA, gelatin, and core-shell fibers with crosslinked mats was analyzed by FTIR spectrometer (Thermo Scientific Nicolet 380). FTIR spectra were obtained within the range of 4000 to $500 \mathrm{~cm}^{-1}$ using 64 scans with a resolution of $2 \mathrm{~cm}^{-1}$.

Water contact angle measurements were performed on crosslinked specimens in air with a sessile-drop method using a goniometer (KSV-CAM 101) and deionized water. At least five measurements from different points were taken and then average values were calculated.

DSC measurements were conducted (Seteram Instrumentation Labsys Evo) using $10 \mathrm{mg}$ samples under nitrogen flow with $10^{\circ} \mathrm{C} / \mathrm{min}$ heating rate from 25 to $250{ }^{\circ} \mathrm{C}$. Tensile strength and fracture elongation values were measured using universal tensile testing machine (Llyod LF Plus) equipped with a $250 \mathrm{~N}$ load cell. At least seven samples were tested at a relative humidity of $50 \%$ with deformation rate of $3 \mathrm{~mm} / \mathrm{min}$. Specimens were prepared in the form of rectangular strips each having a width $\times$ length of $10 \mathrm{~mm} \times 20 \mathrm{~mm}$. Thickness of the mats were determined individually by optical microscope after placing on a glass plate with paying attention not to destroy samples prior to tests.

In vitro degradation test were performed on the enzymatic basis. Crosslinked samples were cut into $50 \mathrm{~mm} \times 50 \mathrm{~mm}$ squares; initial weights were recorded and placed inside plates. About $0.01 M$ PBS containing $0.1 \mathrm{mg} / \mathrm{mL}$ of lysozyme were added into each plate. Samples were placed inside an incubator at $37^{\circ} \mathrm{C}$, for $24,48,72$, and $336 \mathrm{~h}$. At $24 \mathrm{~h}$ intervals, they were rinsed with distilled water and solution re-fed. Samples were then rinsed with distilled water, dried, and weighted to find out degradation rate due to lysozyme enzymatic reaction. The percentage weight loss were calculated as

$$
w \%=\frac{w_{j}-w_{f}}{w_{1}} \times 100
$$

where $w \%$ is the percentage loss and $w_{i}, w_{f}$ are initial and final weight of the samples.

\section{RESULTS AND DISCUSSION}

\section{Morphology of the Electrospun Fibers}

SEM images of electrospun fibers are shown in Figure 2. Pure gelatin fibers exhibited beaded morphology as seen in Figure 2(A) with non-beaded fiber parts having a thickness less than $50 \mathrm{~nm}$. Other fiber diameter values are listed in Table 1 , including pure gelatin $(1 \mathrm{G})$, which did not have a uniform fiber thickness and indicated excess amount of beads. While the diameter of the fibers increased with increasing PVA content, 1P4G exhibited beaded morphology and a broad thickness distribution (Figure $\underline{3}$ ). FRR did not have a significant effect on the fiber diameter, and the fiber diameters were around $300 \mathrm{~nm}$. However, a closer inspection of data in Table 2 reveals a slight increase of the fiber diameter with FRR. In the same table, all groups have closer average pore sizes on the surface except for 1P case. Average pore size of core-shell groups decreased with the increase in the content of gelatin. Determining $1 \mathrm{G}$ surface pore size is hard to achieve with conventional image processing tools. Therefore it was left as not applicable in Table $\underline{1}$. 

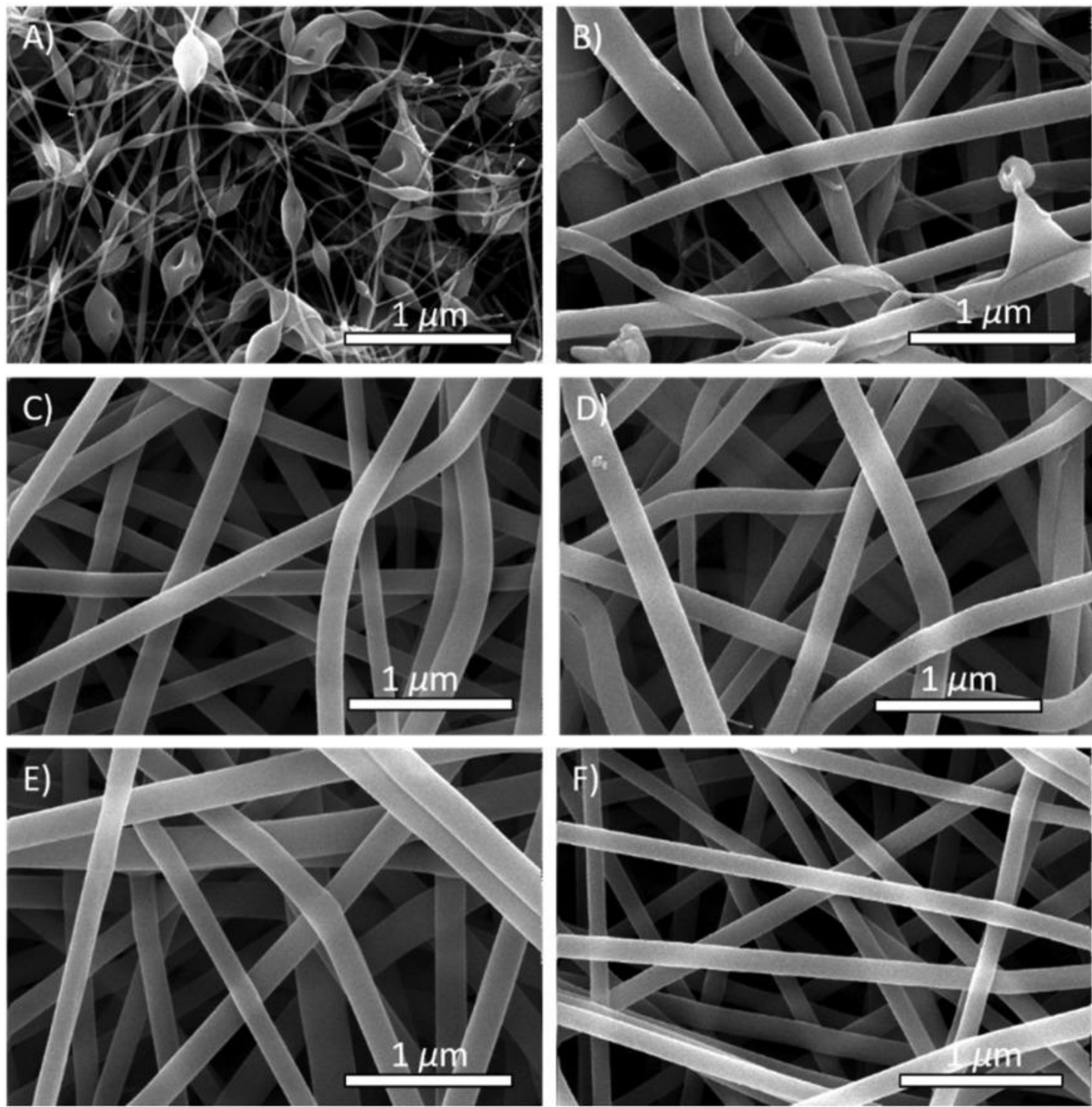

Figure 2 SEM images of the core-shell fibers with different feed ratios of PVA/gelatin. (A) Pure gelatin, (B) 1P4G, (C) 1P2G, (D) 1P1G, (E) 4P1G, (F) pure PVA.

Table 1. Average Fiber Diameter Size, Pore Size, Standard Deviations, and Water Contact Angle Values of Samples

\begin{tabular}{|l|l|l|l|l|l|l|}
\hline \multicolumn{1}{|c|}{ FRR $\rightarrow$} & \multicolumn{1}{c|}{$\mathbf{1 P}$} & \multicolumn{1}{c|}{ 4P1G } & \multicolumn{1}{c|}{ 1P1G } & \multicolumn{1}{c|}{ 1P2G } & \multicolumn{1}{c|}{ 1P4G } & \multicolumn{1}{c|}{ 1G } \\
\hline Fiber diameter $(\mathrm{nm})$ & $200 \pm 40$ & $307 \pm 66$ & $281 \pm 45$ & $282 \pm 66$ & $220 \pm 130$ & $<50$ \\
\hline Pore size $(\mathrm{nm})$ & $586 \pm 595$ & $877 \pm 798$ & $839 \pm 803$ & $703 \pm 701$ & $650 \pm 609$ & NA \\
\hline $\begin{array}{l}\text { Water contact angle } \\
\left(^{\circ}\right)\end{array}$ & $47.0 \pm 2.0$ & $70.0 \pm 3.0$ & $60.0 \pm 5.0$ & $63.0 \pm 4.0$ & $65 \pm 10$ & $41 \pm 10$ \\
\hline
\end{tabular}

\begin{tabular}{|c|l|l|l|l|l|l|l|}
\hline \multicolumn{2}{|c|}{ Sample ID } & 1P & 4P1G & 1P1G & 1P2G & 1P4G & 1G \\
\hline Feed Rates (mL/h) & PVA & 0.1 & 0.4 & 0.1 & 0.1 & 0.1 & - \\
\hline & Gelatin & - & 0.1 & 0.1 & 0.2 & 0.4 & 0.1 \\
\hline
\end{tabular}




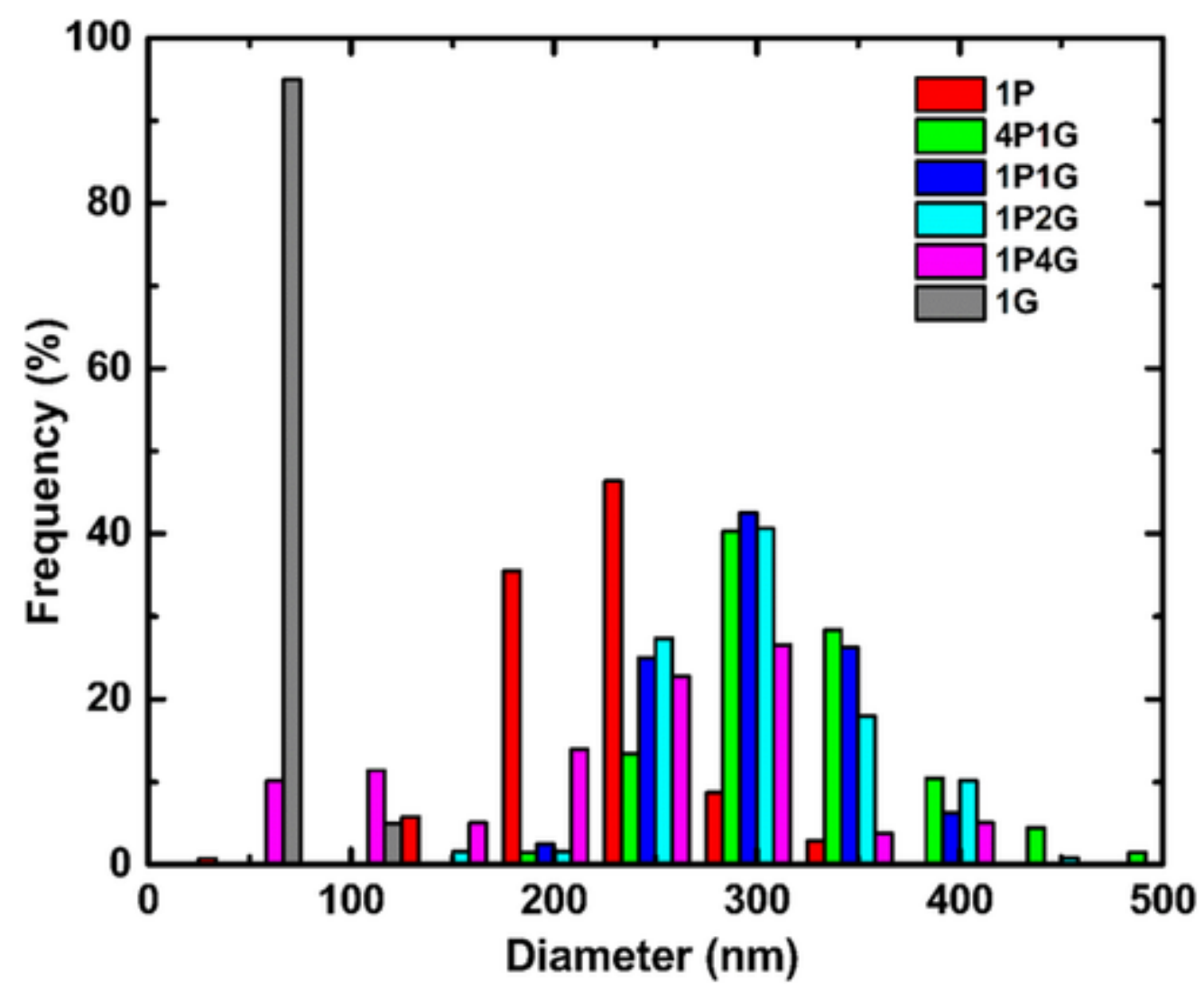

Figure 3 Histogram showing fiber diameter distribution for pure PVA, gelatin, and PVAgelatin core-shell nanofibers.

TEM images in Figure $\underline{4}$ of two different fibers of core-shell structure reflect the general morphological characteristics. To obtain TEM micrograph, potassium permanganate was used to enhance contrast by benefiting from the different affinities of gelatin and PVA on metals. 25
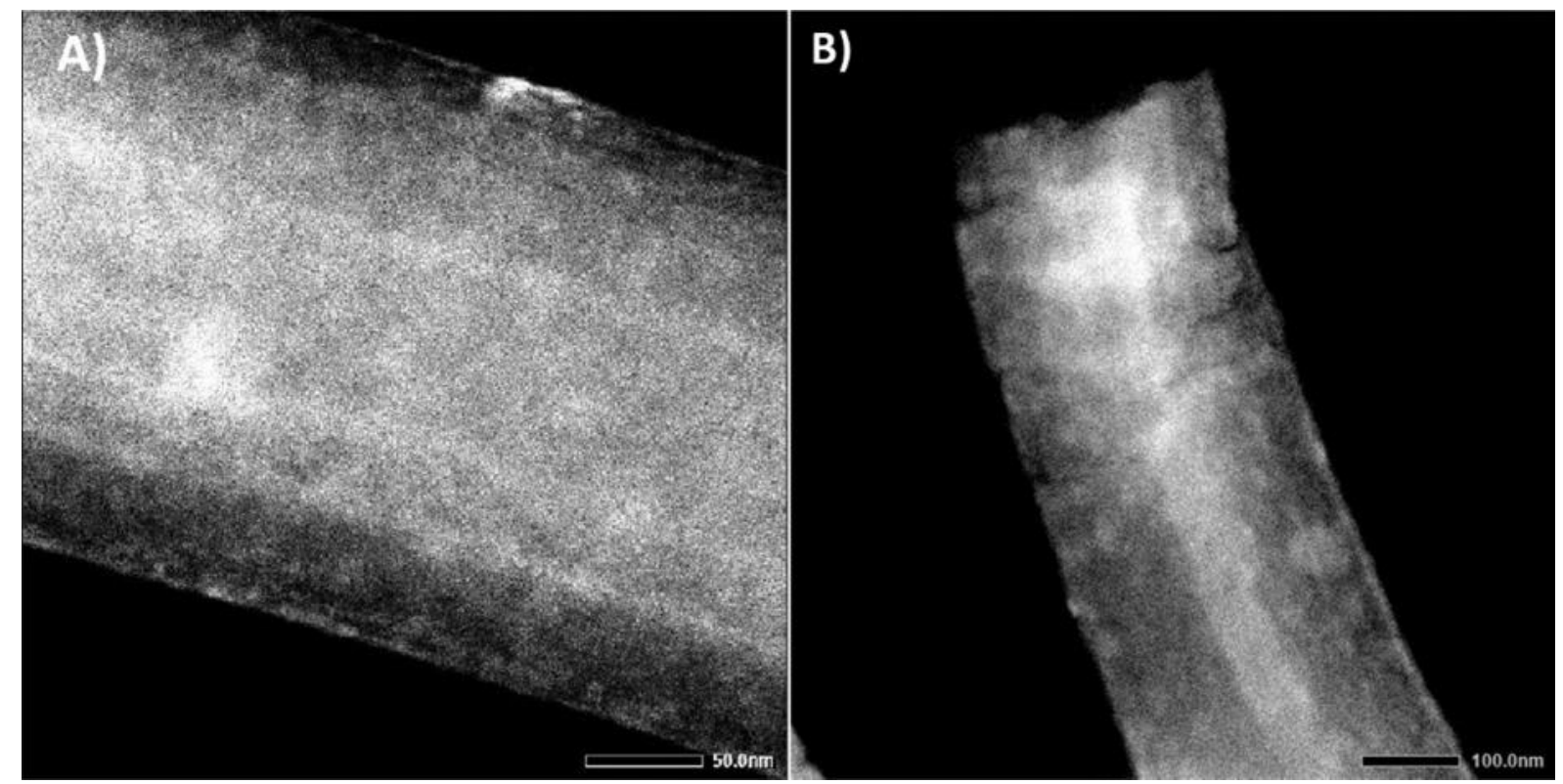

Figure 4 STEM images of two different coaxially electrospun core-shell fibers of PVA/gelatin obtained from $8 \%$ PVA and $8 \%$ gelatin aqueous solutions (FRR 1:1). 
Cells can attach on the hydrophilic surfaces, it is apparent that crosslinking did not change hydrophilic nature of the membranes $\left(<90^{\circ}\right)$ (Table 1 ) There is a close relation between average fiber diameter and specific surface area; the two quantities are inversely proportional. It is clear from the data that decreased fiber diameters resulted in greater wettability. It was previously demonstrated that increased surface area enhances intrinsic wettability properties of nanofibrous substrates.26 For example, increasing the specific surface area of nanofibrous membranes made from hydrophobic polymers results in enhanced hydrophobicity. 26 In contrast, when the same membrane was chemically modified to present hydrophilic moieties at the surface, hydrophilic behavior increases with surface area.27 In the present study, since both PVA and gelatin both exhibited hydrophilic properties due to their polar functional groups, an increase in wettability was expected with decreasing diameter. The increase in fiber diameters in the case of core-shell fibers therefore resulted in a small decrease in wettability.

Concentration of the polymer solution has considerable effect in determining the size of the fabricated nanofibers. $\underline{28}$ Therefore, solutions with minimum possible concentrations were prepared. Although less concentrated solutions usually lead to beaded fibers as in the case of $1 \mathrm{G}$, combining it with a non-beaded polymer inside the core at the controlled FRR leads to uniform fibers. In 1P4G case, gelatin absorption capacity of core fiber was passed which directs to the peeling of outer layer (Figure $\underline{5}$ ). Figure $\underline{5}$ also reveals the core structure of the manufactured fibers.

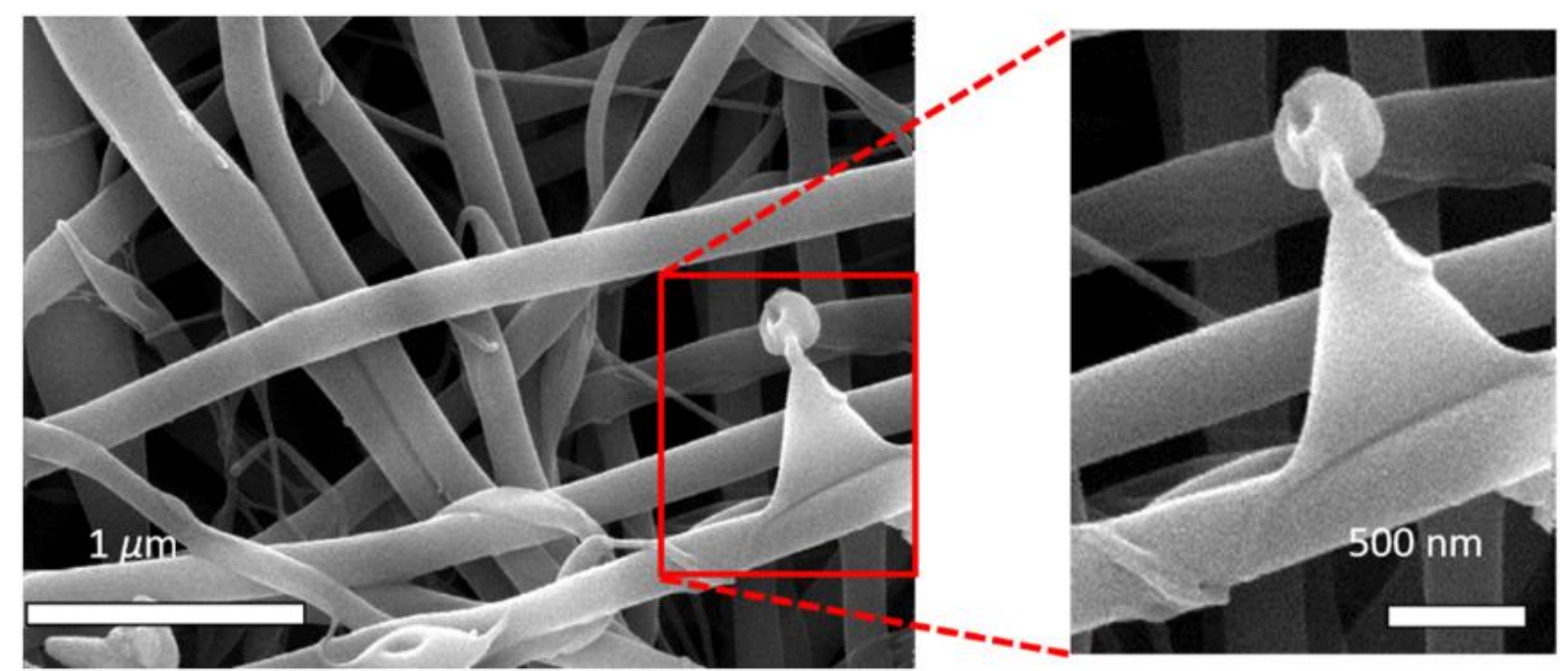

Figure 5 SEM image of 1P4G sample, peeled nanofiber reveals core-shell morphology.

Due to a mismatch in evaporation rates, use of different solvents for core and shell results in porous surfaces with deficiencies in fiber morphology, which necessitate extra optimization steps to minimize abnormalities in morphology. Using co-solvent approach, as adopted in this study, is one of the greener ways to produce core-shell fiber mats and also has the obvious advantage of avoiding toxic residues. $\underline{29}$

\section{Mechanical Properties}

Mechanical properties of the core-shell and 1P mats for crosslinked and non-crosslinked variants are listed in Table $\underline{3}$. Pure gelatin $(1 \mathrm{G})$ fiber mats were excluded since they contain discontinuous fibers, which made extraction from the aluminum foil impossible without damaging. Tensile strength and elongation at fracture values found in literature for non- 
crosslinked $1 \mathrm{G}$ are $3.7 \pm 0.5 \mathrm{MPa}$ and $15 \pm 3.0 \%$ respectively. 30 For crosslinked $1 \mathrm{G}$, the two values were $0.48 \pm 0.20 \mathrm{MPa}$ and $4.3 \pm 0.5 \%, \underline{31}$ respectively. Although those values were obtained at different strain rates and fabrication conditions, they were presented here for comparison purposes. Mechanical results of the remaining cases mostly present a significant decrease in the elongation properties while slight improvements can be seen in tensile strengths after crosslinking.

Table 3. Mechanical Properties of the Crosslinked and Non-crosslinked Groups

\begin{tabular}{|l|l|l|l|l|}
\hline & \multicolumn{2}{|c|}{ Non-crosslinked } & \multicolumn{2}{c|}{ Crosslinked } \\
\hline FRR & $\begin{array}{l}\text { Percent elongation } \\
\text { before failure (\%) }\end{array}$ & $\begin{array}{l}\text { Ultimate tensile } \\
\text { strength (MPa) }\end{array}$ & $\begin{array}{l}\text { Percent elongation } \\
\text { before failure (\%) }\end{array}$ & $\begin{array}{l}\text { Ultimate tensile } \\
\text { strength (MPa) }\end{array}$ \\
\hline 1P & $93.5 \pm 39.0$ & $2.3 \pm 0.3$ & $89.3 \pm 7.50$ & $3.1 \pm 0.2$ \\
\hline 4P1G & $137.4 \pm 12.5$ & $3.9 \pm 0.2$ & $77.5 \pm 5.0$ & $4.8 \pm 0.8$ \\
\hline 1P1G & $80.2 \pm 9.0$ & $2.4 \pm 0.3$ & $28.9 \pm 1.2$ & $4.3 \pm 0.5$ \\
\hline 1P2G & $83.0 \pm 1.1$ & $2.8 \pm 0.2$ & $23.9 \pm 12.7$ & $3.9 \pm 0.6$ \\
\hline 1P4G & $114.0 \pm 15.3$ & $2.9 \pm 0.1$ & $25.4 \pm 3.5$ & $2.3 \pm 0.2$ \\
\hline
\end{tabular}

Gelatin shell increased the strength of pure PVA, which is related to the semicrystal structure of PVA. 31 Alignment of PVA molecules during ES resulted in higher tensile properties.

Crosslinking improved the mechanical properties of the 1P1G samples most, nearly twofolds (from 2.4 to $4.3 \mathrm{MPa}$ ). 1P4G samples slightly lost their mechanical strength due to crosslinking which can be related to the entanglement level that elevates localized stresses in addition to the free forms of short gelatin beaded fibers. $\underline{32}$

\section{FTIR Analysis}

FTIR spectra of the mats were taken to observe secondary structures of gelatin and to observe effect of the crosslinking. Figure $\underline{6}$ shows spectra of as received gelatin powder, crosslinked and non-crosslinked electrospun gelatin, electrospun PVA, and one representative group of core-shell fibers (1P1G). Crosslinking almost had no detectable effect on FTIR spectra in any of the samples, which implies that secondary structures are mostly preserved.

Considering pure gelatin, the peak at $3305 \mathrm{~cm}^{-1}$ can be attributed to amide A and the peak at $3065 \mathrm{~cm}^{-1}$ to amide B. Also, characteristic gelatin peaks observed at 1643, 1536 and 1238 $\mathrm{cm}^{-1}$ are attributed to amide I, amide II and amide III, respectively. ES increased ordered conformations of gelatin as observed by the shift of the amide I peak from 1630 to 1643 $\mathrm{cm}^{-1}$. This shift indicates conversion of beta turn structures to more stable alpha helices. $\underline{33}$ Since ES operates at elevated temperatures, a multi nozzle system was used to complete the process in a short time to preserve the mentioned structure from thermal degradation. The FTIR spectra in Figure $\underline{6}$ (B) show all of the gelatin containing electrospun samples possess the same amide I peak at $1643 \mathrm{~cm}^{-1}$. Comparison of the spectra of crosslinked and noncrosslinked PVA containing specimens has shown $\mathrm{O} \square \mathrm{H}$ stretching of $\mathrm{CH}_{2}$ at $3000-3500$, 1733 , and $2930 \mathrm{~cm}^{-1}$. 

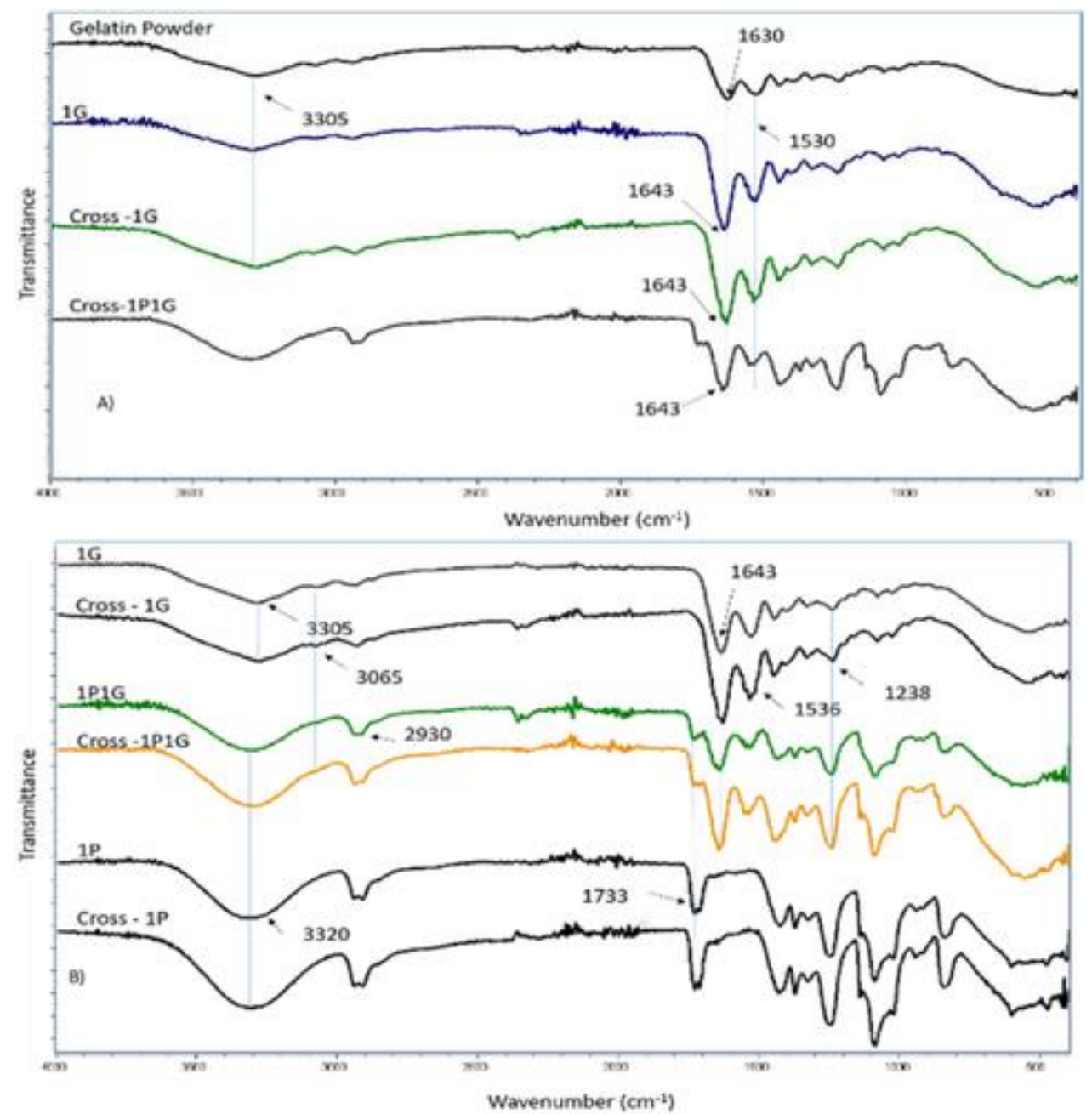

Figure 6 (A) FTIR spectra of gelatin powder before ES, 1G, crosslinked gelatin, crosslinked 1P1G. (B) FTIR spectra of 1G, crosslinked gelatin, core-shell 1P1G, crosslinked 1P1G, 1P, and crosslinked PVA.

\section{Thermal Properties}

Non-crosslinked and crosslinked of selected three groups are presented in Figure $\underline{7}$.

Endothermic peaks at around $\sim 75^{\circ} \mathrm{C}$ for all gelatin containing groups can be attributed to helix to coil transition zone.34 Also in the same zone, water associated evaporation enthalpy difference has considerable impact.34 $1 \mathrm{P} 1 \mathrm{G}$ as an intermediate group in between PVA and gelatin shows characteristics of both polymers separately. It carries melting peak of PVA and denaturation peak of gelatin. $\underline{35}$

Melting and denaturation temperature for all groups were given in Table $\underline{3}$. Results have shown that as gelatin content increased in core-shell samples, $T_{d}$ shifted toward pure gelatin peak (from 69 to $75^{\circ} \mathrm{C}$ ). Melting temperature does not show any appreciable difference.

Miscibility of two polymers can be detected by the merge of melting points. 36 A closer look at plot of non-crosslinked groups (Figure 7), one can detect that $1 \mathrm{P} 1 \mathrm{G}$ case shows both melting temperature characteristics, although in Table $\underline{4}$ only one of them is listed. Also crosslinking smoothened the curves and shifted denaturation temperatures of gelatindominated samples to the right. 37 


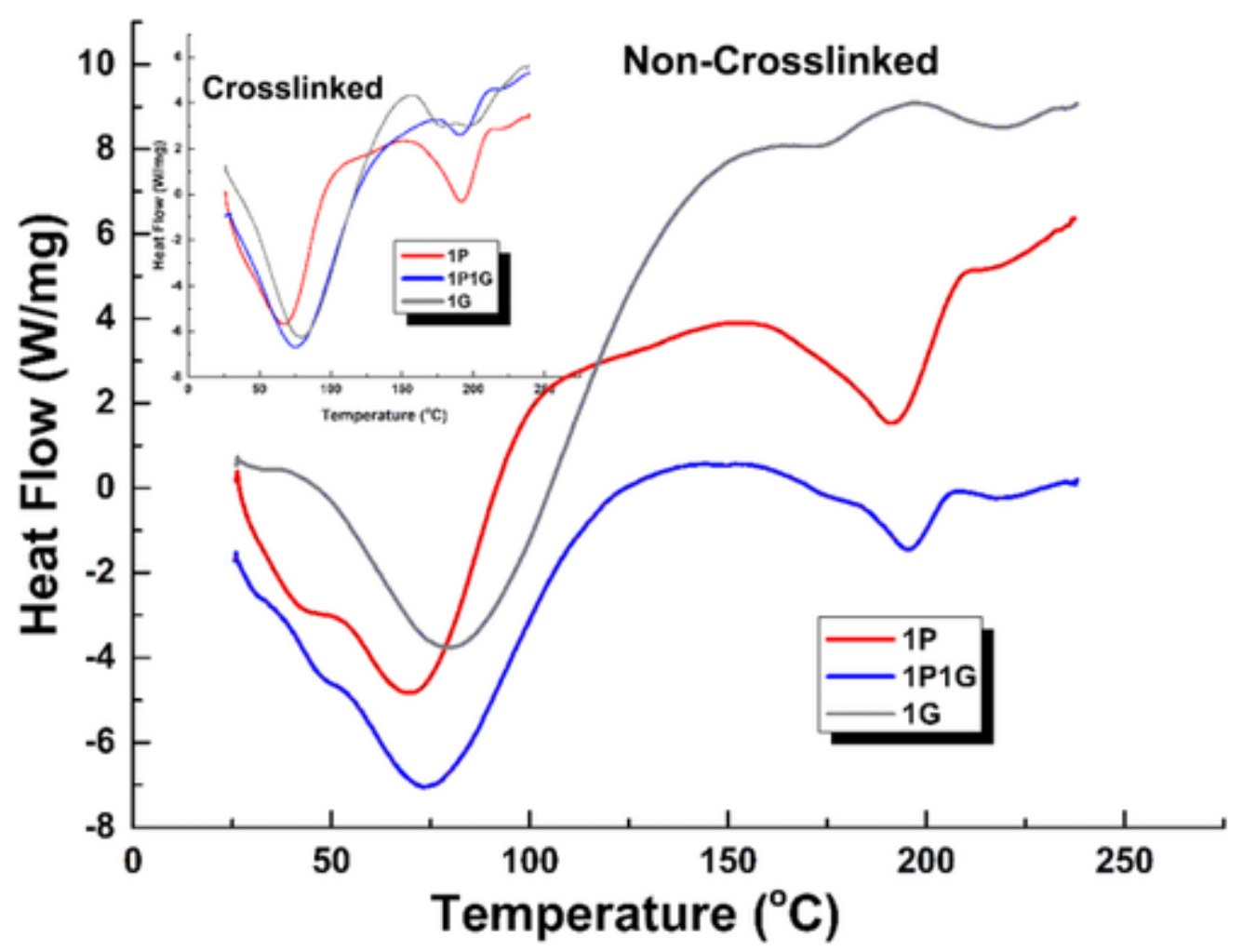

Figure 7 DSC curves of PVA, gelatin, and 1P1G samples

Table 4. Melting $\left(T_{m}\right)$ and Denaturation $\left(T_{d}\right)$ Temperatures of Crosslinked and Noncrosslinked (Normal) Groups Obtained via DSC Measurements

\begin{tabular}{|l|l|l|l|l|}
\hline & \multicolumn{2}{|c|}{ Non-crosslinked } & \multicolumn{2}{c|}{ Crosslinked } \\
\hline & \multicolumn{1}{|c|}{$\boldsymbol{T}_{\boldsymbol{d}}$} & \multicolumn{1}{|c|}{$\boldsymbol{T}_{\boldsymbol{m}}$} & \multicolumn{1}{|c|}{$\boldsymbol{T}_{\boldsymbol{d}}$} & \multicolumn{1}{c|}{$\boldsymbol{T}_{\boldsymbol{m}}$} \\
\hline 1P & 69.93 & 191.6 & 64.76 & 192.1 \\
\hline 4P1G & 71.65 & 195.41 & 68 & 189.82 \\
\hline 1P1G & 74.3 & 195.68 & 75.12 & 192.7 \\
\hline 1P2G & 76.43 & 195.32 & 76.9 & 192.9 \\
\hline 1P4G & 75.16 & 195.14 & 82.57 & 194.22 \\
\hline 1G & 79 & 219.8 & 78.93 & 197 \\
\hline
\end{tabular}

- Note: For $1 \mathrm{P} T_{d}$ is dehydration temperature.

\section{In Vitro Degradation}

As shown in the degradation tests (Figure $\underline{8}$ ), $1 \mathrm{G}$ had the least degradation after 14 days (16\%). Main reason might be due to that the fiber diameters are below $50 \mathrm{~nm}$ resulted much more surface area for crosslinking than other groups that have $\sim 6$ times larger diameter. Approximately, $80 \%$ of $1 \mathrm{P}$ group degraded in the first day. In addition to the surface area effect, gelatin has more and many groups (free amino, hydroxyl groups) that can be bind by glutaraldahyde, $\underline{38}$ but PVA has only one group to bind (hydroxyl). $\underline{39}$ This might be one reason for rapid degradation that is observed in $1 \mathrm{P}$ case. Degradation rates of core-shells, generally, decrease as the gelatin rate increased. Also, core-shell morphology can be used to control degradation rates of composite polymers, as the present study reveals different FRRs have different degradation times. In addition, increased fiber diameters and increased 
porosity levels have significant impact on degradation. Former one reduces the surface to be crosslinked, latter one allows lysozyme to reach voluminous regions.

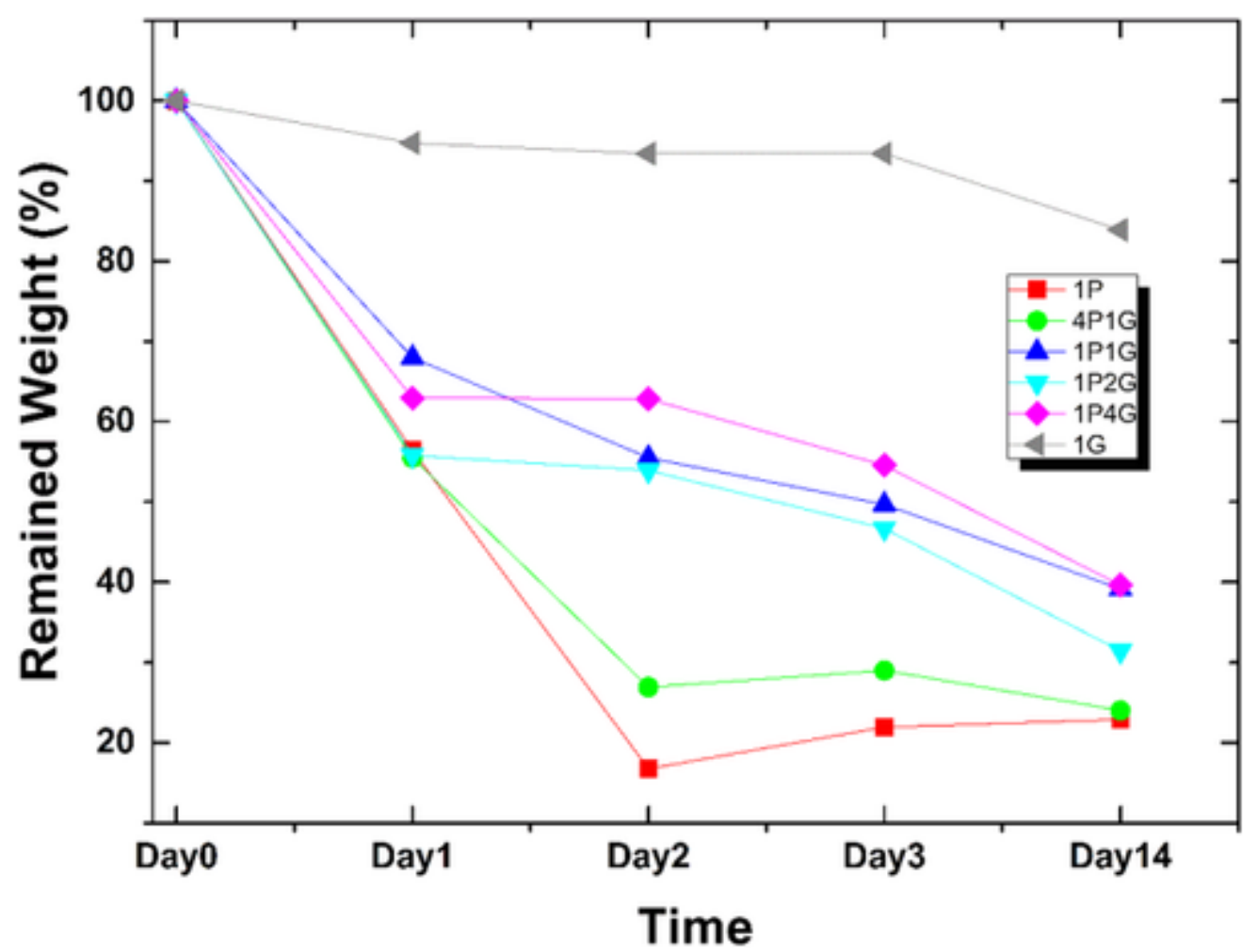

Figure 8 In vitro enzymatic degradation of crosslinked as-spun samples for 14 days.

\section{CONCLUSIONS}

Non-toxic solvents enhance biocompatibility of the fibers and reduce the risk of cytotoxicity. In this study, different characterization methods were used to confirm core-shell morphology of the fibers. Varied FRRs showed that fiber diameter decrease as gelatin ratio increases below the limiting ratio of 1:4 (PVA:gelatin) at the prescribed ES parameters. Water contact angle measurement results supported hydrophilic nature of the gelatin-covered samples after crosslinking. FTIR data revealed the ordering effect of ES on the secondary structures inside gelatin. Tensile tests showed that crosslinking increases ultimate tensile strength and thermal properties showed that. Hence, PVA-gelatin core-shell scaffolds will be potential candidates for tissue engineering and biomedical applications, uniting presence of cell recognition sites with mechanical endurance.

\section{ACKNOWLEDGMENTS}

First author would like to thank Amitav Sanyal for kind helps, Bilge Gedik for SEM measurements, and Işıl Yeşil for FTIR analysis. This work is partially supported by Bogazici University Research fund by Grant number no: 6701. TEM analysis leading to these results has received support by the Nanotechnology Research and Application Center at Sabanci University. Authors would like to thank Central Research Laboratory, Ordu University for DSC measurements. 